\section{OVERWEIGHT OR OVERWEIGHT WITH BODY FAT DISTRIBUTION? WHAT PREDICT BEST HORMONAL, METABOLIC AND INFLAMMATORY PROFILES IN CHILDREN?}

H. Samouda ${ }^{1}$, C. de Beaufort ${ }^{2}$, U. Schierloh ${ }^{2}$, J. Jacobs ${ }^{3}$, G. Gilson ${ }^{4}$, M. Vaillant ${ }^{3}$, F. Dadoun ${ }^{5}$, M.L. Lair ${ }^{3}$, OSPEL study group

${ }^{1}$ Department of Public Health, Centre de Recherche Publique-Santé, Strassen, ${ }^{2}$ DECCP, Clinique Pédiatrique/CHL, ${ }^{3}$ Department of Public

Health, Centre de Recherche Publique-Santé, ${ }^{4}$ Department of Clinical Biology, ${ }^{5}$ Departement de Diabétologie/Endocrinologie, Centre Hospitalier de Luxembourg, Luxembourg, Luxembourg

Objective: To analyze the additional effect of body fat distribution anthropometric indexes to BMI Z Score for assessing hormonal, metabolic and inflammatory profiles amongst overweight and obese children.

Research design and methods: 203 overweight and obese children (7-17 years, 106 girls, 97 boys) from the OSPEL study participated in anthropometric and biological assessment with personal and parental informed consent.

Results: After adjustement for sex and age, prediction of fasting insulin, HOMA IR, QUICKI, triglycerides, HDL cholesterol, CRP and adiponectin was improved when combining Waist to Hip Ratio $Z$ Score with BMI Z Score. Likewise, fasting insulin, triglycerides, HDL Cholesterol, SBP Z Score, CRP and adiponectin were best predicted by the conicity index-BMI Z Score association. Anthropometric prediction of fasting insulin, HOMA IR, QUICKI, triglycerides, HDL cholesterol and resistin was also improved when the Kahn index and the BMI Z Score were associated. WHR Z Score, Conicity and Kahn indexes were independently and significantly associated to the multivariate prediction models. According to metabolic syndrome (MetS) definition used [IDF (1) versus Cook/Ford (2)], the increase by one unit of WHR Z Score (respectively the increase by 0.1 unit of Conicity index) multiplies the risk for developing MetS, from 2.4 to 8, for comparable BMI $Z$ Score values.

Conclusions: Body fat distribution, beyond overweight and obesity, should be taken into account to predict cardiovascular risk profile in children and adolescents.

\section{RESULTS FROM THE MERENDA CROSS OVER TRIAL: EVALUATING THE EFFECT OF BRAND ON AD LIBITUM SNACKING AFTER SCHOOL FREE TIME}

\author{
D. Gregori ${ }^{1}$, L. Franchin², MERENDA Study Group \\ ${ }^{1}$ Department of Environmental Medicine and Public \\ Health, University of Padova, Padova, ${ }^{2}$ ZETA \\ Research Ltd, Trieste, Italy
}

Introduction: Snacking is suspected to have an association with overweight and obesity in children, due to the higher caloric intake of snacks, which consumption is promoted by advertising and branding. Methods: 96 children between 6 and 11 years old have been randomized to three groups: No exposure to TV, Exposure to TV and Exposure to TV and advertising. All children were asked to eat ad libitum during the afternoon break a set of sweet snacks. The sample has been stratified according to age, gender and brand awareness, which was assessed via a flash card based instrument. The study was designed to have a 0.80 power to detect a 0.5 SD units difference between either exposure groups and the no-exposure. Total quantity in grams and overall intake in calories has been computed after a 20 minutes time of eating. Analyses have been based on ANCOVA and adjusted by socioeconomic status. Results: Mean (SD) amount eaten and mean (SD) calories intake are shown below.

Low Brand Awareness

Quantity (grams) NoTV:76.5(8) TV:77(10) TV+Advertising:76(10)

Calories NoTV:331(41) TV:337(32) TV+Advertising:338(39)

High Brand Awareness

Quantity (grams) NoTV:78.7(10) TV:80(11) TV+Advertising:77(11)

Calories NoTV:338(37) TV:341(32)

TV+Adertising:334(43)

No significant difference are emerging.

Conclusion: In an ad libitum eating experiment on snacks, awareness of branding does not seem to have an effect on the amount eaten both in terms of caloric intake and of overall quantity. This is also not changing according to TV exposure. 